\title{
RELATIVISTIC CELESTIAL MECHANICS AND REFERENCE FRAMES
}

\author{
T. DAMOUR \\ Institut des Hautes Etudes Scientifiques, 91440 Bures sur Yvette, France and \\ Département d'Astrophysique Relativiste et de Cosmologie, Observatoire de \\ Paris - Centre National de la Recherche Scientifique, 92195 Meudon Cedex, \\ France \\ M. SOFFEL $\dagger$ and C. XU* \\ Theoretische Astrophysik, Universität Tübingen, Auf der Morgenstelle 10, 7400 \\ Tübingen, FRG
}

\begin{abstract}
A new formalism for treating the relativistic celestial mechanics of systems of $N$, arbitrarily composed and shaped, weakly self-gravitating, rotating, deformable bodies is presented. This formalism is aimed at yielding a complete description, at the first post-Newtonian approximation level, of (i) the global dynamics of such $N$-body systems ("external problem"), (ii) the local gravitational structure of each body ("internal problem"), and, (iii) the way the external and the internal problems fit together ("theory of reference systems").
\end{abstract}

\section{Introduction}

The problem of describing the dynamics of $N$ gravitationally interacting extended bodies, called "celestial mechanics", has been thoroughly investigated (see e.g. Tisserand, 1960) in the framework of Newton's theory of gravity. Very shortly after the discovery of Einstein's theory of gravity, Einstein (1915), Droste (1916), De Sitter (1916) and Lorentz and Droste (1917) devised an approximation method (called "post-Newtonian") which allowed them to compare General Relativity with Newton's theory of gravity, and to predict several "relativistic effects" in celestial mechanics, such as the relativistic advance of the perihelion of planets, and the relativistic precession of the Moon's orbit. This post-Newtonian approach to relativistic celestial mechanics was subsequently developed (and completed) by many authors, notably by Fock (1959), Papapetrou (1951), Chandrasekhar and colleagues

† Research supported by the "Deutsche Forschungsgemeinschaft" and by the "Stiftung Volkswagenwerk". M.S. kindly acknowledges the receipt of a Heisenberg fellowship.

* On leave from Fudan University, Shanghai, P.R. China. 
(1965, 1969, 1970), Caporali (1981), Grishchuk and Kopejkin (1986) and others (for a review of the development of the problem of motion in General Relativity see e.g. Damour, 1987).

However, in order to match the high precision which is already achieved by means of space techniques such as Satellite Laser Ranging (SLR), Lunar Laser Ranging (LLR) or Very Long Baseline Interferometry (VLBI), one needs a correpondingly accurate relativistic theory of celestial mechanics able to describe both the global gravitational dynamics of a system of $N$ extended bodies, the local gravitational structure of each, arbitrarily composed and shaped, rotating deformable body, and the way each of these $N$ local structures meshes into the global one. The traditional post-Newtonian approach to relativistic celestial mechanics using only one global coordinate system $x^{\mu}=(c t, x, y, z) \equiv\left(c t, x^{i}\right), i=1,2,3$, to describe an $N$-body system, fails in this task, for both conceptual and technical reasons; concepts like "center of mass", "multipole moments", "mass-centered frames" are used while they are ill defined. Usually such "mass-centered frames" with spatial coordinates $X^{i}$, e.g. given by

$$
X^{i}=x^{i}-z^{i}(t)
$$

where $z^{i}$ denutes the global coordinates of the "center of mass" of the body under consideration are not dynamically useful in the sense that they do not efface the external gravitational field down to tidal effects but, instead, introduce into the description of the internal dynamics of the bodies many external "relativistic" effects proportional to the square of the orbital velocity or the external gravitational potential. This is because the external (global) description of each body contains many "apparent deformations" (Lorentz contractions etc.) which are not intrinsic to the body itself.

In recent years, several authors have tried to remedy some of the defects of the traditional post-Newtonian approach to the $N$-body problem. For instance, Martin et al. (1985) and Hellings (1986) have tried, in an essentially heuristic manner, to explicitly take into account the main apparent deformations due to the use of an external coordinate representation. More recently a notable progress in the theory of such local relativistic frames (at the post-Newtonian approximation, relevant to systems of $N$ weakly self-gravitating bodies) has been achieved by Brumberg and Kopejkin (1988a,b) (Kopejkin, 1988; Brumberg, 1990) in a series of publications (see also Voinov, 1988). Their approach combines the usual post-Newtonian-type expansions with the multipole expansion formalisms for internally generated (Thorne, 1980; Blanchct and Damour, 1986, 1989) and externally generated (Thorne and Hartle, 1985), gravitational fields, and with asymptotic matching techniques (D'Eath, 1975, Damour, 1983). We believe, however, that the approach by Brumberg and Kopejkin has several drawbacks: ad hoc assumptions about the structure of various expansions (as e.g. in the coordinate transformation between global and local coordinates) are made, which are only partially justified by some later consistency checks; the scheme is confined to a particular model for the matter (isentropic perfect fluid) 
and rigidly restricts itself to considering only some special (harmonic) coordinate system; moreover, their approach is basically incomplete in that it neither describes the full multipole moment structure of the bodies with post-Newtonian accuracy, nor gets (translational or rotational) equations of motion with full post-Newtonian accuracy.

\section{A New Approach Towards Relativistic Celestial Mechanics}

We have introduced (Damour, Soffel and $\mathrm{Xu}, 1990 \mathrm{a}$ ) a new formalism for treating the relativistic celestial mechanics of systems of $N$, arbitrarily composed and shaped, weakly self-gravitating, rotating, deformable bodies. This formalism yields a complete description, at the first post-Newtonian level, of the global dynamics of such $N$-body systems ("external problem"), the local gravitational structure of each body ("internal problem"), and the way they fit together ("relativistic theory of reference sysiems"). This new scheme successfully overcomes, in our opinion, the problems encountered by previous approaches (notably the one of Brumberg and Kopejkin): only very general assumptions are made for the structure of the formalism which is developed in a constructive way by proving a number of theorems; the structure of the stress-energy tensor of the matter is left completely open; the scheme is formulated in a certain "gauge-invariant" way which leaves a convenient flexibility in the choice of the time gauge (at the order $\delta t=O\left(c^{-4}\right)$ ); the scheme describes with full post-Newtonian accuracy the gravitational structure of each body by means of a set of multipole moments which are linked in an operational way to what can be observed in the local gravitational environment of each body; finally, the scheme succeeds in getting translational and rotational equations of motion with full post-Newtonian accuracy, and inclusion of all multipole moments, for the $N$-body system. Our approach does not use any asymptotic matching technique but takes advantage of two different recent progresses in the first post-Newtonian approximation method: (i) linearization of Einstein's field equations by means of certain "exponential parametrization" of the metric tensor (introduced by Blanchet and Damour (1989), and Blanchet, Damour and Schäfer (1990)), and (ii) definition, by Blanchet and Damour (1989), of a set of post-Newtonian multipole moments of an isolated body given as compact support integrals of the stress-energy tensor of the matter. A third basic element of the present approach is our way of restricting (without fixing completely) the coordinate freedom inherent to the theory of General Relativity. We do that not by imposing one of the two differential coordinate conditions generally used in the post-Newtonian literature (namely "harmonic gauge" versus "standard post-Newtonian gauge") but by imposing, in all coordinate systems, some algebraic conditions on the metric coefficients, which can be written as $(i, j=1,2,3)$

$$
g_{00} g_{i j}=-\delta_{i j}+O\left(1 / c^{4}\right)
$$


This condition can be described by saying that the spatial coordinates are "conformally cartesian" or "isotropic". This condition is compatible with both usual choices but is, at once, more flexible (for the time gauge) and more rigid (for the space gauge) than either one of them. It plays an important technical role in freezing down the coordinate freedom to a level which is nearly the usual freedom in Newtonian celestial mechanics (arbitrary choice of a time-dependent spatial origin and of a time-dependent rotation matrix).

\section{Theory Of Reference Systems}

For our problem of $N$ gravitationally interacting extended bodies we employ $N+1$ coordinate charts (reference systems): one "global" chart with coordinates $x^{\mu}=$ $\left(c t, x^{i}\right)$ and $N$ "local" charts with coordinates $X^{\alpha}=\left(c T, X^{a}\right)$. Each one of the local charts is defined in the vicinity of some body, and comoving with it. For most practical purposes in the solar system two of these coordinate system will be sufficient: one global "barycentric" system $\left(c t, x^{i}\right)$ and one local "geocentric" system $\left(c T, X^{a}\right)$. The global, barycentric coordinate time $t$ is also named TCB, whereas the geocentric coordinate time $T$ also is called TCG.

In each of these reference systems we use an exponential representation for the metric tensor of the form

$$
\begin{aligned}
& g_{00}=-e^{-2 w / c^{2}}+O(6) \\
& g_{0 i}=-\frac{4}{c^{3}} w_{i}+O(5) \\
& g_{i j}=+e^{+2 w / c^{2}} \gamma_{i j}+O(4)
\end{aligned}
$$

where $O(n) \equiv O\left(c^{-n}\right)$ indicates the post-Newtonian order of magnitude. In this expression $w$ is a generalization of the usual Newtonian potential and $w^{i}$ is some gravitational vector potential arising because of "magnetic type gravity". One finds that the Riemann curvature tensor of $\gamma_{i j}$ is of order $O(4)$, i.e. to post-Newtonian $(\mathrm{PN})$ order the space metric is conformally flat. Hence, there exists a preferred class of spatial coordinates, where

$$
\gamma_{i j}=\delta_{i j}+O(4)
$$

or, equivalently, where condition (2) holds. In the formulation of our framework we systematically use such preferred spatially isotropic coordinates. Then, for each reference system, the information in the metric tensor is fully contained in the scalar field $w$ and the vector field $w_{i}$. The Einstein field equations remarkably become linear in terms of these variables:

$$
\begin{aligned}
\Delta w+\frac{3}{c^{2}} \partial_{t}^{2} w+\frac{4}{c^{2}} \partial_{t i}^{2} w_{i} & =-4 \pi G \sigma+O(4) \\
\Delta w_{i}-\partial_{i j}^{2} w_{j}-\partial_{t i}^{2} w & =-4 \pi G \sigma^{i}+O(2),
\end{aligned}
$$


where

$$
\begin{aligned}
\sigma & =\frac{T^{00}+T^{s s}}{c^{2}} \\
\sigma^{i} & =\frac{T^{0 i}}{c}
\end{aligned}
$$

Though, for each reference system, our spatial coordinates are fixed (modulo a choice of origin and rigid rotation) by our spatial isotropy condition, we do not fix completely our time coordinate, but keep a certain flexibility linked to a gauge invariance of the $1 \mathrm{PN}$ field equations: if $w_{\mu} \equiv\left(w, w_{i}\right)$ is a solution of eqs.(5) with some given source terms $\sigma^{\mu} \equiv\left(\sigma, \sigma^{i}\right)$ so is $w_{\mu}^{\prime}=\left(w^{i}, w_{i}^{\prime}\right)$ (modulo PN error terms) with

$$
\begin{aligned}
w^{\prime} & =w-\frac{1}{c^{2}} \partial_{t} \lambda, \\
w_{i}^{\prime} & =w_{i}+\frac{1}{4} \partial_{i} \lambda
\end{aligned}
$$

where $\lambda\left(x^{\mu}\right)$ is an arbitrary (differentiable) function. This gauge invariance corresponds to a shift of the time variable according to

$$
\delta t=\frac{1}{c^{4}} \lambda(t, x)
$$

which affects none of the physical quantities at the 1PN level. This especially applies to the problem of time scales: the relation between barycentric coordinate time ( $t=$ TCB) and geocentric coordinate time $(T=\mathrm{TCG})$ is not affected by transformation (8) to post-Newtonian order. Note, that our gauge freedom encompasses both the choice of the "harmonic gauge" as well as of the "standard post-Newtonian gauge". E.g., for the harmonic gauge the solution of the field equations reads

$$
\begin{aligned}
w & =G I_{-1}[\sigma]+\frac{G}{2 c^{2}} \partial_{t}^{2} I_{1}[\sigma]+O(4) \\
w_{i} & =G I_{-1}\left[\sigma^{i}\right]+O(2),
\end{aligned}
$$

where

$$
I_{\alpha}[f](t, x) \equiv \int d^{3} x^{\prime}\left|x-x^{\prime}\right|^{\alpha} f\left(t, x^{\prime}\right) .
$$

Similarly to what is done in Maxwell's theory of electromagnetism, we can introduce gauge-invariant (gravito-electric and gravito-magnetic) fields $e$ and $b$ by

$$
\begin{aligned}
\boldsymbol{e} & \equiv \nabla \boldsymbol{w}+\frac{4}{c^{2}} \partial_{t} \boldsymbol{w} \\
\boldsymbol{b} & \equiv-4 \nabla \times \boldsymbol{w}
\end{aligned}
$$


satisfying (in each system) "Maxwell-like" equations of the form

$$
\begin{aligned}
\nabla \cdot \boldsymbol{b} & =0 \\
\nabla \times \boldsymbol{e} & =-\frac{1}{c^{2}} \partial_{t} b \\
\nabla \cdot \boldsymbol{e} & =-\frac{3}{c^{2}} \partial_{t}^{2} w-4 \pi G \sigma+O(4), \\
\nabla \times \boldsymbol{b} & =4 \partial_{t} \boldsymbol{e}-16 \pi G \sigma+O(2) .
\end{aligned}
$$

For the coordinate transformation between each of the local coordinates $X^{\alpha}$ and the global coordinates $x^{\mu}$ we start with the completely general ansatz

$$
x^{\mu}=f^{\mu}\left(X^{\alpha}\right)=z^{\mu}\left(X^{0}\right)+e_{a}^{\mu}\left(X^{0}\right) X^{a}+\xi^{\mu}\left(X^{0}, X^{a}\right)
$$

Here, $a=1,2,3$ labels the spatial coordinates in the local system, $z^{\mu}\left(X^{0}\right)\left(X^{a}=0\right)$ describes the "global" motion of some "central worldline" of the body under consideration (which will later be chosen as the worldline of the "center of mass" of the body) and $\xi^{\mu}$ is assumed to be at least quadratic in $X^{a}$. Now, our PN assumptions plus spatially isotropic coordinates essentially determine $f^{\mu}\left(X^{\alpha}\right)$ completely, modulo the choices of some arbitrary central worldline and of some (slowly varying) rotation matrix $R_{a}^{j}$ in $e_{a}^{i}(T)$ (see eq.(23) below). E.g., one finds uniquely

$$
\xi^{i}(T, X)=\frac{1}{c^{2}} e_{a}^{i}(T)\left[\frac{1}{2} A_{a} X^{2}-X^{a}(A X)\right]+O(4)
$$

where

$$
A_{a} \equiv f_{\mu \nu} e_{a}^{\mu} \frac{d^{2} z^{\nu}}{d T^{2}}
$$

(the 4-acceleration of the central worldline projected into the corresponding local system). Here, $f_{\mu \nu}$ denotes the usual flat Minkowski metric in Cartesian coordinates $\left(f_{\mu \nu}=\operatorname{diag}(-1,+1,+1,+1)\right)$.

Because of the linearity of the field equations, in each local system, we can uniquely split the metric potentials $\dagger W_{\alpha} \equiv\left(W, W_{\alpha}\right)$ into "self-" and "external-part"

$$
W_{\alpha}=\stackrel{+}{W}_{\alpha}+\bar{W}_{\alpha}
$$

Here, the self-part $\left(W^{+}{ }_{\alpha}\right)$ describes the gravitational influence of the central body itself, while the external-part describes the action of all the other bodies of the system (plus inertial terms).

$\dagger$ We use capital letters for local quantities. 
As central results of our scheme we find the following transformation rules of potentials between some local and the global system to PN order:

$$
\begin{aligned}
w & =\left(1+\frac{2}{c^{2}} V^{a} V^{a}\right) W+\frac{4}{c^{2}} V^{a} W_{a}+\frac{c^{2}}{2} \ln \left[A_{0}^{0} A_{0}^{0}-A_{a}^{0} A_{a}^{0}\right] \\
w^{i} & =R_{a}^{i} W^{a}+v^{i} W+\frac{c^{3}}{4}\left[A_{0}^{0} A_{0}^{i}-A_{a}^{0} A_{a}^{i}\right]
\end{aligned}
$$

where

$$
A_{\alpha}^{\mu} \equiv \frac{\partial x^{\mu}}{\partial X^{\alpha}}
$$

and $v^{i}$ is the velocity of the "central point" in the global system $\left(V^{a}=R_{i}^{a} v^{i}\right)$. Hence, not only the field equations are linear, but also the various $w \leftrightarrow W$ relationships! Writing this affine transformation in the form

$$
w^{\mu}(x)=\mathcal{A}_{\alpha}^{\mu}(T) W^{\alpha}(X)+\mathcal{B}^{\mu}(X)
$$

we find the transformation of the self-parts to take the simple form

$$
\stackrel{+}{w}^{\mu}(x)=\mathcal{A}_{\alpha}^{\mu}(T) \stackrel{+}{W}^{\alpha}(X)
$$

a remarkable result indeed. Hence, in contrast to previous works on relativistic reference systems, we obtain the various transformation laws in closed, i.e., nonexpanded form (we do not use a matched asymptotic expansion technique like, e.g, Brumberg and Kopejkin), which has in fact many advantages for practical applications.

We introduce the following (BD) mass $\left(M_{L}\right)$ and current moments $\left(S_{L}\right)$ of the central body defined by ( $L$ is a multi spatial index, $L \equiv a_{1} \ldots a_{l}$ )

$$
\begin{aligned}
M_{L}= & \int d^{3} X \hat{X}_{L} \Sigma+\frac{1}{2(2 l+3) c^{2}} \frac{d^{2}}{d t^{2}}\left[\int d^{3} X \hat{X}_{L} X^{2} \Sigma\right] \\
& -\frac{4(2 l+1)}{(l+1)(2 l+3) c^{2}} \frac{d}{d t}\left[d^{3} X \hat{X}_{a L} \Sigma_{a}\right], \\
S_{L}= & \int d^{3} X \epsilon^{a b<c_{l}} \hat{X}^{L-1>a} \Sigma^{b}
\end{aligned}
$$

where all quantities are considered in the local system of the considered body, where the caret and the bracket $\langle>$ indicates that the symmetric and trace-free (STF) part should be taken (see e.g. Thorne, 1980) and the integration extends over the support of the body under consideration. These BD-moments are called "physical" by us because the self-part of the local gravitational potentials of the considered 
body can be expanded in terms of these multipole moments (modulo an irrelevant gauge transformation). See Blanchet and Damour (1989) for the other physical meanings of these moments when considering isolated systems.

Not only are the self-potentials $W^{+}{ }_{\alpha}$ expanded in terms of (STF) moments, but similarly the external-potentials $\bar{W}_{\alpha}$ or the corresponding $\bar{E}$ and $\bar{B}$-fields are "skeletonized" by defining (for each local system) two corresponding (gravito-electric and gravito-magnetic) sets of post-Newtonian tidal moments:

$$
\begin{aligned}
& (l \geq 1) \quad G_{L} \equiv\left[\partial_{<L-1} \bar{E}_{a_{l}>}(T, X)\right]_{X^{a}=0}, \\
& (l \geq 1) \quad H_{L} \equiv\left[\partial_{<L-1} \bar{B}_{a_{l}>}(T, X)\right]_{X^{a}=0} .
\end{aligned}
$$

Using the various expansions of the self-potentials and the transformation laws we can get the external tidal moments explicitly as functions of the intrinsic moments $M_{L}, S_{L}$ of all the other bodies, plus some inertial contributions.

If we require (as we may) the quantities $e_{\alpha}^{\mu}\left(e_{0}^{\mu} \equiv c^{-1} d z^{\mu}(T) / d T\right)$ to represent an orthonormal tetrad with respect to the "external metric" defined by $\bar{w}_{\mu}$, our theory of reference systems is completely specified up to the choice of:

- the time gauge

- the central worldlines, $z^{i}(T)$

and a slowly time dependent rotation matrix $R_{a}^{j}(T)$ appearing in

$$
e_{a}^{i}(T)=\left(1-\left.\frac{1}{c^{2}} \bar{w}\right|_{X^{a}=0}\right)\left(\delta^{i j}+\frac{1}{2 c^{2}} v^{i} v^{j}\right) R_{a}^{j}(T) .
$$

\section{A User's Guide To Reference Systems}

For practical purposes let us summarize our results for relativistic reference frames for the problem of barycentric and geocentric coordinate systems. The global, barycentric coordinate system was denoted by $\left(c t, x^{i}\right)$, the local geocentric one by $\left(c T, X^{a}\right)$. The barycentric (geocentric) coordinate time $t(T)$ is also called TCB (TCG). The relation between the barycentric and the geocentric system is written in the form (13). For many practical applications presently one can neglect the quadratic and higher order terms $\xi^{\mu}$ in (13); then the relation between these coordinate systems is simply given by:

$$
\begin{aligned}
c t & =z^{0}(T)+e_{a}^{0}(T) X^{a} \\
x^{i} & =z^{i}(T)+e_{a}^{i}(T) X^{a} .
\end{aligned}
$$


For the relation between $t=\mathrm{TCB}$ and $T=$ TCG one finds the result:

$$
\left.\left.\frac{d t}{d T}\right|_{X^{a}=0} \equiv \frac{d(\mathrm{TCB})}{d(\mathrm{TCG})}\right|_{X^{a}=0}=e_{0}^{0}=1+\frac{1}{c^{2}}\left(\bar{w}\left(z_{\oplus}\right)+\frac{1}{2} v_{\oplus}^{2}\right) \text {. }
$$

Here, $X^{a}=0$ refers to the geocenter, $v_{\oplus}$ is the velocity of the geocenter in the barycentric system and $\bar{w}$ can be replaced by the external Newtonian potential $U_{\text {ext }}$ taken at the geocenter. For the linear term in the time transformation $e_{a}^{0}(T)$ in eq.(24a) one finds to sufficient approximation

$$
e_{a}^{0} \simeq R_{a}^{j} v_{\oplus}^{j}
$$

In this last formula the matrix $R_{a}^{j}$ relates the spatial barycentric coordinates with the spatial geocentric ones, which not necessarily point into the same direction. Using these results the TCB-TCG relation can be written in the form

$$
\mathrm{TCB}-\mathrm{TCG}=c^{-2} \int_{t_{0}}^{t}\left(\frac{1}{2} \boldsymbol{v}_{\oplus}^{2}+U_{\mathrm{ext}}\left(\boldsymbol{z}_{\oplus}\right)\right) d t+\boldsymbol{v}_{\oplus} \cdot \boldsymbol{X}
$$

Here, $v_{\oplus} \cdot X=v_{\oplus}^{j} R_{a}^{j} X^{a} \simeq v_{\oplus}^{j}\left(x^{j}-z_{\oplus}^{j}\right)$

To post-Newtonian accuracy the spatial coordinates $x^{i}$ and $X^{a}$ are related by

$$
x^{i}=z_{\oplus}^{i}(T)+e_{a}^{i} X^{a}+O\left(X^{2}\right)
$$

where $z_{\oplus}^{i}(T)$ describes the motion of the geocenter and the coefficients $e_{a}^{i}$ are determined by eq.(23) which we may write in the form

$$
e_{a}^{i}(T)=\left(1-\frac{1}{c^{2}} U_{\mathrm{ext}}\left(z_{\oplus}\right)\right)\left(\delta^{i j}+\frac{1}{2 c^{2}} v_{\oplus}^{i} v_{\oplus}^{j}\right) R_{a}^{j}(T) .
$$

The matrix $R_{a}^{j}(T)$ finally determines the precise relation between the barycentric and the geocentric spatial coordinates. Two choices for $R_{a}^{j}(T)$ are preferred, leading. to geocentric coordinates which are

- fixed star oriented (kinematically non-rotating)

- or locally inertial (dynamically non-rotating).

In the first case of kinematically non-rotating geocentric coordinates we might take

$$
R_{a}^{j}(T)=\delta_{i j}
$$

In this case the direction of geocentric spatial coordinates are given by the corresponding directions of barycentric coordinates, i.e. practically by some catalogue of extragalactic sources. If such kinematically non-rotating geocentric coordinates are 
chosen then additional (time dependent) inertial forces, mainly due to the geodesic precession (of inertial axes) have to be taken into account in any dynamical equation (e.g. for an artificial satellite). The geodesic precession in this case has to be considered also in the precession matrix as well as in the nutation series (because the geodesic precession has an annual term proportional to the eccentricity of the Earth's orbit).

On the other hand, if dynamically non-rotating, locally inertial geocentric coordinates are chosen the geodesic precession is contained in the $R_{a}^{j}(T)$ matrix and not in the precession (nutation) matrix. This choice, however, has the disadvantage, that the local geocentric spatial coordinate lines precess w.r.t. the barycentric ones.

\section{Equations of Motion}

In our approach, global equations of motion are derived by combining the local energy-momentum balance equations

$$
T_{\alpha ; \beta}^{\beta}=0
$$

with conditions chosen to relate the central worldline of a body with the corresponding energy-momentum distribution. We find that a theorem of the following form holds in each local frame

Theorem. The energy-momentum conservation equations (24) in each local frame imply constraints on the time-evolution of the three lowest $B D$ multipole moments of the form:

$$
\begin{aligned}
& \frac{d M}{d T}=\frac{1}{c^{2}} \mathcal{E}^{1 \mathrm{PN}}\left(\stackrel{(p)}{M}{ }_{L}, \stackrel{(p)}{G}_{L^{\prime}}\right)+O(4), \\
& \frac{d^{2} M_{a}}{d T^{2}}=\sum_{l \geq 0} \frac{1}{l !} M_{L} G_{a L}+\frac{1}{c^{2}} \mathcal{F}_{a}^{(1 \mathrm{PN})}\left(\stackrel{(p)}{M} L, \stackrel{(q)}{S}{ }_{L} ; \stackrel{(p)}{G}_{L^{\prime}},{\stackrel{\left(q^{\prime}\right.}{H}}_{L^{\prime}}\right) \\
& +O(4) \\
& \frac{d S_{a}}{d T}=\sum_{l \geq 0} \frac{1}{l !} \epsilon_{a b c} M_{b L} G_{c L}+\frac{1}{c^{2}} \mathcal{G}_{a}^{(1 \mathrm{PN})}\left(\stackrel{(p)}{M}_{L}, \stackrel{(q)}{S}_{L} ;{\stackrel{\left(p^{\prime}\right)}{G}}_{L^{\prime}},{\stackrel{\left(q^{\prime}\right.}{H}}_{L^{\prime}}\right) \\
& +O(2 / 4)
\end{aligned}
$$

where

$$
\stackrel{(p)}{M} \equiv \frac{d^{p}}{d T^{p}} M \quad \text { etc. }
$$


and all the right-hand sides of eqs.(31) are bilinear in the BD multipole moments and in the above-introduced tidal moments, and their time derivatives.

More explicitly, the right-hand sides of eqs.(31) consist of an infinite series of terms, each having the form

$$
\stackrel{(p)(q)}{M} G, \stackrel{(p)(q)}{M} \stackrel{(p)(q)}{H}, \stackrel{\text { or }}{G}, \quad \stackrel{(p)(q)}{S} \stackrel{H}{H}
$$

The special notation $O(2 / 4)$ in eq.(31c) means that, when one is working strictly within the 1PN approximation, it is sufficient to know $S_{a}$ to Newtonian accuracy and therefore the explicitly written Newtonian torque is enough. However, it is possible to define a local spin vector for body $A$ (differing from the Newtonian spin moment $(21 \mathrm{~b})$ by $O\left(c^{-2}\right)$ additional terms) whose time evolution is given, modulo $O(4)$, by an equation of the form (31c).

To relate the central worldline of a body with the corresponding energy-momentum distribution, we choose each central worldline to coincide with the BD center of mass of the considered body, i.e., we require $M_{a}=0$. From eq.(31b) this then implies

$$
0=\frac{d^{2} M_{a}}{d T^{2}}=\sum_{l \geq 0} \frac{1}{l !} M_{L} G_{L a}+\left(c^{-2}-\text { terms }\right) .
$$

Since one can prove that

$$
G_{a}=-\frac{d^{2} z^{a}(t)}{d t^{2}}+\left.\bar{w}_{, a}\right|_{X^{a}=0}+\left(c^{-2}-\text { terms }\right)
$$

we see that the "local equation of motion" (32) can be rewritten in the looked for global form for the equation of translational motion:

$$
\frac{d^{2} z^{a}(t)}{d t^{2}}=\left.\bar{w}_{, a}\right|_{X a=0}+\sum_{l \geq 2} \frac{1}{l !} M_{L} G_{L a} / M+\left(c^{-2}-\text { terms }\right)
$$

where we have derived the complete PN expression on the right hand side of eq.(34) for arbitrary mass- and current-moments of the individual bodies (Damour, Soffel and $\mathrm{Xu}, 1990 \mathrm{~b}$ ). We have explicitly verified (Damour, Soffel and Xu, 1990a) that in the monopole limit without spins ("spherical, non-rotating" bodies) one recovers the usual Lorentz - Droste - Einstein - Infeld - Hoffmann equations of motion used for modern numerical ephemeris programs (such as the DE programs from JPL). Work for the PN-spin motion is still in progress. 


\section{References}

Blanchet, L., and Damour, T., 1986, Phil. Trans. R. Soc. London, A320, 379

Blanchet, L., and Damour, T., 1989, Ann. Inst. Henri Poincaré 50, 377

Blanchet, L., Damour, T., and Schäfer, G., 1990, Mon. Not. R. astr. Soc. 242, 289

Brumberg, 1990, Essential Relativistic Celestial Mechanics, Hilger, Bristol, to be published

Brumberg, V.A., and Kopejkin, S.M., 1988a, in: Reference Systems, eds. J. Kovalvsky, I.I. Mueller and B. Kolaczek, Reidel, Dordrecht

Brumberg, V.A., and Kopejkin, S.M., 1988b, Nuovo Cim B, 103, 63

Caporali, A., 1981, Nuovo Cimento B 61, 181, 205 and 213

Chandrasekhar, S., 1965, Astrophys. J. 142, 1488;

Chandrasekhar, S., and Nutku, Y., 1969, Astrophys. J. 158, 55

Chandrasekhar, S., and Esposito, F., 1970, Astrophys. J. 160, 153

Damour, 'T., 1983, in: Gravitational Radiation, ed. N. Deruelle and T. Piran (Les Houches 1982), pp. 59-144, North-Holland, Amsterdam

Damour, T., 1987, in: 300 Years of Gravitation, ed. S.W. Hawking and W. Israel, Cambridge University Press, Cambridge

Damour, T., Soffel, M., and Xu, C., 1990a, to be submitted for publication

Damour, T., Soffel, M., and Xu, C., 1990b, to be submitted for publication

D'Eath, P.D., 1975, Phys. Rev. D11, 1387

De Sitter, W., 1916, Mon. Not. R. Astr. Soc., 76, 699; 77, 155

Droste, J., 1916, Versl. K. Akad. Wet. Amsterdam, 19, 447

Einstein, A., 1915, Preuss. Akad. Wiss. Berlin, Sitzber., p.831, 18 November 1915

Fock, V., 1959, The Theory of Space, Time and Gravitation, Pergamon Press, Oxford 
Grishchuk, L., and Kopejkin, S.M., 1986, in: Relativity in Celestial Mechanics and Astrometry, ed. J. Kovalevsky and V. Brumberg, Reidel, Dordrecht

Hellings, R.W., 1986, Astron. J., 91, 650

Kopejkin, S.M., 1988, Celest. Mech. 44, 87

Lorentz, H.A., and Droste, J., 1917, Versl. K. Akad. Wet. Amsterdam, 26, 392 (part I) and 649 (part II). English translation in H.A. Lorentz, Collected Papers, ed. P. Zeeman and A.D. Fokker, vol. V, pp. 330-55. Martinus Nijhoff: The Hague (1937)

Martin, C.F., Torrence, M.H., and Misner, C.W., 1985, J. of Geophys. Res., 90, 9403

Papapetrou, A., 1951, Proc. R. Soc. London A 209, 248

Thorne, K.S., 1980, Rev. Mod. Phys., 52, No. 2, Part I, 299

Thorne, K.S., and Hartle, J.B., 1985, Phys. Rev., D31, 1815

Tisserand, F., 1960, Traité de Mécanique Céleste, vol.I, Gauthier-Villars: Paris (reprint of the first edition of 1889)

Voinov, A.V., 1988, Celest. Mech., 42, 293 